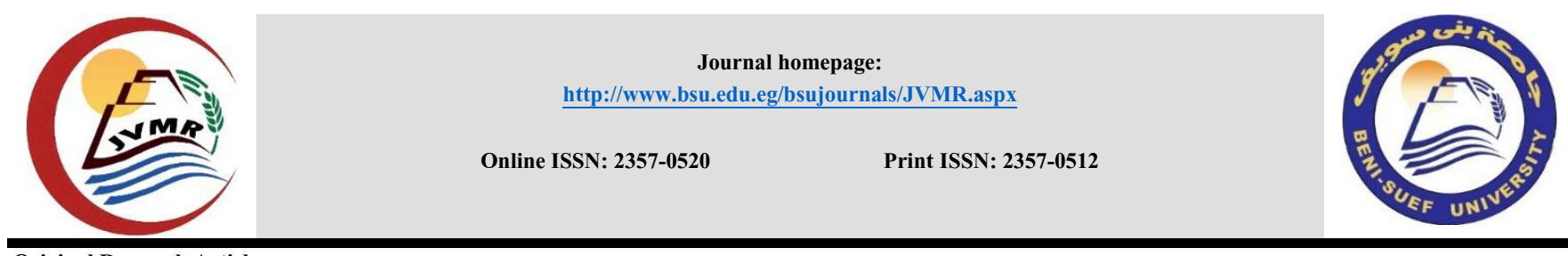

Original Research Article

\title{
Pathogenicity of Escherichia coli 0125 in commercial broiler chickens
}

\author{
Asmaa Abd Elatiff ${ }^{1}$, Azza A. El-Sawah ${ }^{2}$, Mohamed M. Amer ${ }^{3}$,Al-Hussein M. Dahshan ${ }^{2}$, Hala Salam ${ }^{4}$, Salama A.S.Shany ${ }^{2}$ \\ ${ }^{1}$, Faculty of agriculture, E1 Minya University . \\ ${ }^{2}$ Poultry Diseases, Faculty of Veterinary Medicine, Beni-Suef University, Beni-Suef 62511, Egypt. \\ ${ }^{3}$ Poultry Diseases, Faculty of Veterinary Medicine, Cairo University ,Giza12618, Egypt \\ ${ }^{4}$ Bacteriology, Mycology and Immunology Disease, Faculty of Veterinary Medicine, Beni-Suef University.
}

\begin{tabular}{|c|c|}
\hline ABSTRACT & ARTICLE INFO \\
\hline $\begin{array}{l}\text { Commercial broiler chickens are frequently infected with Ecoli serotypes in form of collibacillosis which } \\
\text { characterized as an initial respiratory infection (air sacculitis) followed by generalized septicemia, perihepatitis and } \\
\text { pericarditis. The present work aimed to study the ability of E.coli O125 previuosly isolated and identified from } \\
\text { diseased broilers suffered from pericarditis, airsacculitis and perihapatitis to induce a disease and The current study } \\
\text { aimed to determine the pathogenicity of E. coli serogroup O125 in broiler chicks, a trial of prevention and treatment } \\
\text { using probiotic ( Guardyazin-m) or antibiotic (Cipronate } 20 \% \text { ). The chicks were divided into seven groups including } \\
\text { control negative, orally infected, subcutenously infected, probiotic treated group, and finally antibiotic treated } \\
\text { group. The clinical signs, mortality, performance parameters and postmortem examination as well as the } \\
\text { pathological changes in liver and intestine were recorded. The probiotic group gave the best results in controlling } \\
\text { infection by E. coli O125 followed by antibiotic. In conclusion, the E. coli O125 serogroup was pathogenic to chicks } \\
\text { when experimentally inoculated, so particular attention must be directed toward E. coli O125 as a pathogen infecting } \\
\text { bird. Also probiotics was of great value in protection against the E. coli infection and improve the performance } \\
\text { parameters of chicks . Its effect on feed consumption, weekly body weight gain and feed conversion rate (FCR) was } \\
\text { determined. }\end{array}$ & $\begin{array}{l}\text { Broilers } \\
\text { E. coli } \\
\text { Pericarditis } \\
\text { FCR }\end{array}$ \\
\hline
\end{tabular}

\section{Introduction}

E. coli infection in poultry is one of the principle causes of mortality and morbidity in chickens and turkeys resulting in significant economical losses due to, retardation of growth and decreased feed conversion rate, The infection is aggravated by poor hygiene in breeding farms and faulty management at the hatchery (Kim and Kim, 2010). Other factors that favor rapid bacterial growth include the fact that the yolk contains a lot of fat and water, favored nutrients for bacteria (Shaw and Halvorson, 1993) E. coli infection called collibacillosis (Gomis et al 2001, Bopp et al 2005). The most common form of collibacillosis is characterized as an initial respiratory infection (air sacculitis) in 3-12 weeks old broiler chickens and turkeys, which is frequently followed by generalized septicemia, perihepatitis and pericarditis. E. coli infections include egg peritonitis, omphalitis, coligranuloma, swollen head syndrome, cellulitis, and colisepticaemia. Colisepticaemia is a severe systemic form of infection (Dho Mouline and Fairbrother, 1999; Barnes et al., 2003).

Asmaa (2013) studied the experimental infection with E.coli and the clinical signs appeared in the 1st day post infection in form of dullness, depression white and brown diarrhea in 5 $(20 \%)$ chicks and gradually developed to respiratory signs . The postmortem lesions were septicemic picture from 1 st to 7 th day post infection and intestine filled with yellowish materials then pericarditis and airsaculitis observed at 8-12 day post infection.. probiotics may directly alter the intestinal microbiome, resulting in the same effects. They hypothesized that adding prebiotics and probiotics to feed may protect the gut of young chicks under stress. Studies 1, 2, and 3 evaluated treatments in 
a cold stress (CS) and E coli oral challenge to 430 day-old broiler chicks for $3 \mathrm{wk}$. the probiotic treatment improved production values and might had a potential as an alternative to antibiotics during the first weeks after hatching. Nawaz et al (2016) studied that the efficacy of two probiotics of different origins (yeast and bacterial based) on the growth performance, immune response, carcass characteristics and nutrient digestibility of broilers.

The aim of the present work was to study the effect of the most prevalent E. coli serotype which previously isolated from internal organs and performance of chicks.

\section{Materials and methods}

\subsection{E. coli isolate}

A half milliliter of bacterial suspension of E. coli O125 in sterile saline $(6 \times 108 \mathrm{CFU} / \mathrm{ml}$ adjusted by $2 \mathrm{McFarland} \mathrm{s}$ Barium sulphate standard solution), previously isolated from liver, heart blood and lung specimens of diseased broilers samples, serotyped and detected its virulence via Congo red binding assay and its hemolytic activity.

\subsection{E. coli isolates pathogenicity in day-old chicks}

One strain was selected for pathogenicity testing (O125). Unvaccinated 1-day-old commercial broiler chicks were used. five birds/ isolate were orally and subcutaneously inoculated with $0.5 \mathrm{ml}$ containing $6.0 \times 10^{8} \mathrm{CFU} / \mathrm{ml}$. Birds were maintained for 7 days postinoculation and monitored daily for clinical signs and mortality (Vidotto et al., 1990).

\subsection{Experimental infection test :}

One hundered and fifty arber acerss chicks in (one day old) were used for experimental infection (Table 1) at $14^{\text {th }}$ ages . broth culture of the identified E. coli O125 isolate inoculated by oral and subcutaneous route of infection .Chicks were kept under daily observation for mortality, clinical signs and post mortem picture.

\subsection{Reisolation of the $E$. coli}

Cultures from heart, lung liver and spleen were inoculated in tryptone soya broth and incubated at $37{ }^{\circ} \mathrm{C}$ for $18-24 \mathrm{~h}$. Then loopful from the inoculated broth were streaked into EMB agar and incubated at $37{ }^{\circ} \mathrm{C}$ for $24-48$ h. E. coli was counted on eosin methylene blue (EMB).

\subsection{Treatment schemes in commercial broiler chicks}

After starting of clinical signs treatment were started by using cipronat $20 \%$ (ciprofloxacin) according to our previously study and Probiotic Guardizen $-\mathrm{M}$ which act by competitive exclusion product in drinking water for 7 successive days before the first day of infection batch no F16HO93 . Birds were weighted daily, feed consumption and reisolation from liver, lung and heart blood regularly during experiment. Metal cages were used for grouping of chicks ,commercial balanced ration free from anticoccidials was used for feeding of all chicks groups adlibtum,

\section{Table 1. Total number of chicks used in experiment}

\begin{tabular}{cc}
\hline Group* & Total no. \\
\hline OE & 30 \\
\hline SE & 30 \\
\hline POE & 25 \\
\hline PSE & $\mathbf{2 5}$ \\
\hline C & 25 \\
\hline Pilot experiment & 15 \\
\hline Total & 135 \\
\hline
\end{tabular}

* OE: group infected orally with E. coli O125.- SE: group infected subcutaneous(s/c) -POE: probiotic with orally infected group - PSE: probiotic with $\mathrm{s} / \mathrm{c}$ infected group $-\mathrm{C}$ : control group .

\subsection{Histopathological examination of hepatic and intestinal} sections :

Specimens from intestine and liver were immediately taken after scarification, then fixed in 10\% neutral buffered formalin solution in which they were preserved till processed. Parts from the taken tissue specimens were then washed, dehydrated, embedded in paraffin, sectioned at 4-5 micron thickness and stained with $\mathrm{H} \& \mathrm{E}$ and the power of microscopical examination write on the bar as a routine work for histopathological studies according to Bancroft and Stevens (1996).

\section{Results}

\subsection{Clinical signs and mortality :}

Both control negative group and antibiotic treated group after orally or subcutaneously infection showed no clinical signs and no mortalities, $\boldsymbol{O} \boldsymbol{E}$ started signs at $48 \mathrm{hrs}$. after infection while in $\boldsymbol{S E}$ appeared in 36 hours after infection and the degree of signs in table 2 . These signs were dullness, depression, dropping of wings, off food, ruffled feather and gradually developed to greenish brown diarrhea, at later stage arthritis, ralls, sneezing and coughing. In POE showed no clinical signs till the end of the experiment while $\boldsymbol{P S E}$ the signs appeared as slight dullness, depression, ruffled feather and gradually 
developed to greenish brown diarrhea . At later stage $\left(15^{\text {th }} \mathrm{dpi}\right)$ arthritis, ralls and coughing .

\subsection{Different infected chick's group performance}

Performance parameters measurments revealed that a. control negative group had the best mean weekly body weight (1923.8 \pm 229), weekly feed consumption (1269 g), weekly body weight gain (915 g) and FCR (1.4) at end of experiment $\left(5^{\text {th }}\right.$ week)

b. Orally untreated was significantly lower in weekly body weight $(\mathbf{1 1 5 0 . 4} \pm \mathbf{1 9 6})$ also lower weekly feed consumption (767.7 g), weekly body weight gain (488.5 g) and FCR (1.6) at end of experiment than control negative group which had mean weekly body weight (1923.8 \pm 229), weekly feed consumption (1269 g), weekly body weight gain (915 g) and FCR (1.4) fig (1) and (2) . c. Subcutaneously untreated infected group had significantly lower in weekly body weight (1294.7 \pm 114$)$, also performance parameters showed lower weekly feed consumption (737.6 g), weekly body weight gain (409.8 g) and FCR (1.8) at end of experiment . weekly body weight (1270.7 \pm 618) of orally infection with treatment showed un significant difference with orally infected untreated group and was significantly lower than control negative group at end of experiment, also the performance parameters showed weekly feed consumption (771.7 g), weekly body weight gain (586.8 g) and FCR (1.3) at end of experiment which were relatively better than orally infected untreated group and lower than control negative group.

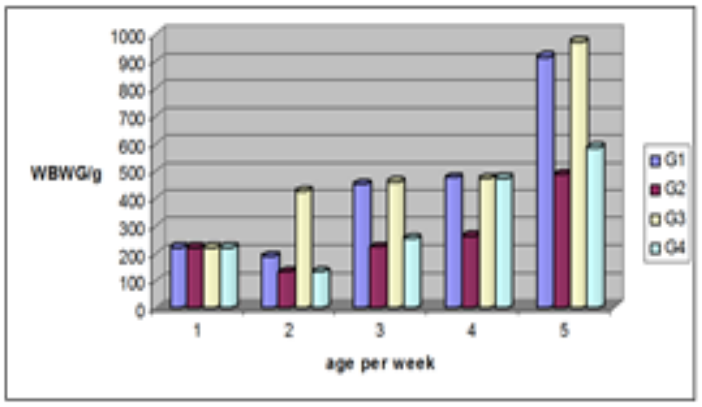

Fig (2): Weekly body weight gain/g in different orally inoculated groups.

Table (2) Estimation of pm lesions degree in all experimental groups :
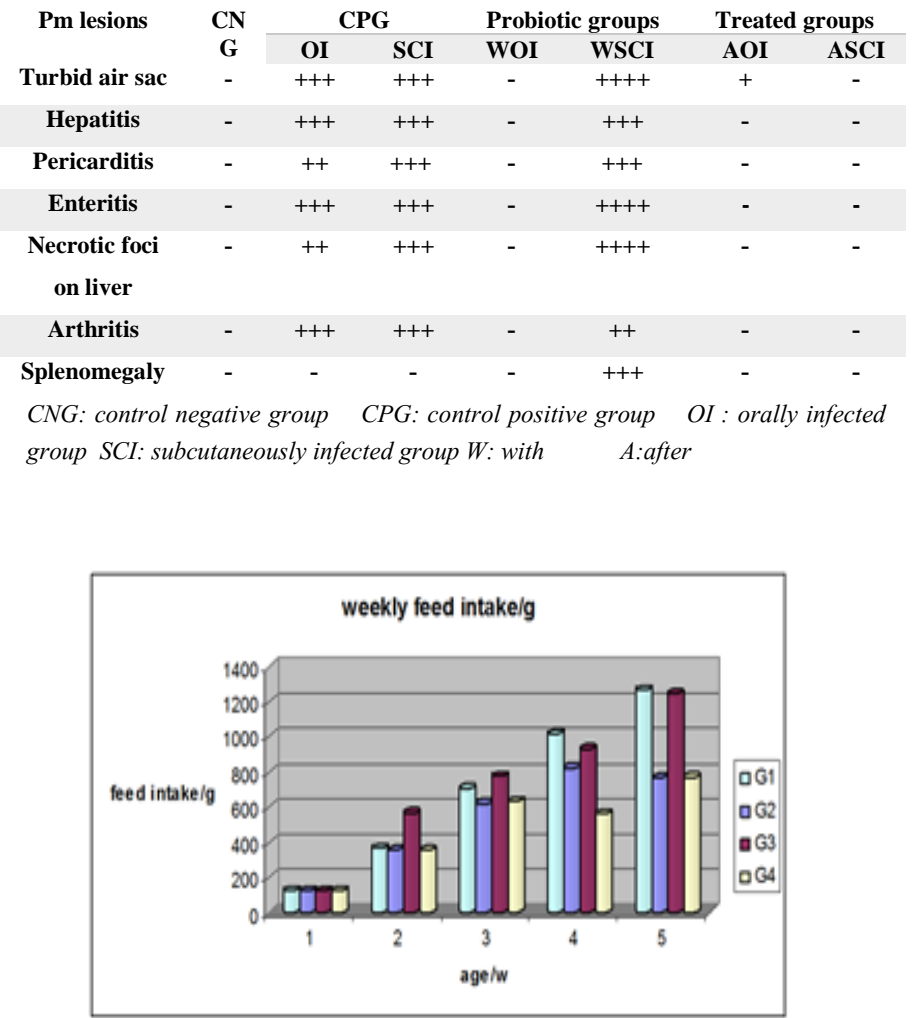

Fig (1) : Weekly feed consumption/g in different orally inoculated groups. (G1-negative control group G2-infected untreated group G3-probiotic treated group before infection G4-Antibiotic treated group after infection).

And the subcutaneously infection with treatment had significantly higher mean weekly body weight $\mathbf{( 1 3 7 9 . 1 5} \pm \mathbf{4 6 5})$ than subcutaneously infected untreated group and significantly lower than control negative group at end of experiment, also the performance parameters showed weekly feed consumption(740 g), weekly body weight gain (528 g) and FCR (1.4) at end of experiment which was better than subcutaneously infected untreated group and lower than control negative group probiotic before orally infection was significantly higher in main weekly body weight $(\mathbf{1 9 0 7 . 3 5} \pm \mathbf{2 1 7 g})$, weekly feed consumption (1247.2g), weekly body weight gain $(\mathbf{9 7 0 . 2 g})$ and FCR (1.3) at end of experiment than probiotic before subcutaneously infection which had mean weekly body weight (1739.9 $\pm \mathbf{2 0 8}$ g), weekly feed consumption (947 g), weekly body weight gain (658.4 g) and FCR (1.4) as shown in Table (4) and Fig. (3) . 
Table (3) Performance parameters of chicks in different subcutaneously inoculated group

W- age per week

G1-negative control group G2-infected untreated group G3-probiotic treated group before infection G4-Antibiotic treated group afterinfection.

\begin{tabular}{|c|c|c|c|c|c|c|c|c|c|c|c|c|c|c|c|c|}
\hline \multirow{4}{*}{$\begin{array}{l}\text { Group } \\
\text { Age }\end{array}$} & \multicolumn{4}{|c|}{$\mathrm{Gl}$} & \multicolumn{4}{|c|}{ G2 } & \multicolumn{4}{|c|}{ G3 } & \multicolumn{4}{|c|}{ G4 } \\
\hline & Body & $\begin{array}{l}\text { Feed } \\
\text { intake }\end{array}$ & $\begin{array}{c}\text { Body } \\
\text { weight } \\
\text { gain }\end{array}$ & \multirow[t]{3}{*}{ FCR } & & $\begin{array}{c}\text { Feed } \\
\text { intake }\end{array}$ & $\begin{array}{c}\text { Body } \\
\text { weight } \\
\text { gain }\end{array}$ & \multirow[t]{3}{*}{ FCR } & Body & $\begin{array}{l}\text { Feed } \\
\text { intake }\end{array}$ & $\begin{array}{l}\text { Body } \\
\text { weight } \\
\text { gain }\end{array}$ & \multirow[t]{3}{*}{ FCR } & Body & $\begin{array}{l}\text { Feed } \\
\text { intake }\end{array}$ & $\begin{array}{c}\text { Body } \\
\text { weight } \\
\text { gain }\end{array}$ & \multirow[t]{3}{*}{ FCF } \\
\hline & weight $(\mathrm{g})$ & (g) & (g) & & \multirow{2}{*}{$\begin{array}{l}\text { weight (g) } \\
\text { Mean } \pm \text { SD }\end{array}$} & \multirow[b]{2}{*}{ (g) } & \multirow[t]{2}{*}{ (g) } & & weight $(g)$ & (g) & $(g)$ & & weight (g) & (g) & (g) & \\
\hline & Mean \pm SD & & & & & & & & Mean $\pm S D$ & & & & Mean \pm SD & & & \\
\hline wl & $91 \pm 81$ & 126.9 & 221.9 & 0.6 & $91 \pm 81$ & 126.9 & 221.9 & 0.6 & $91 \pm 81$ & 126.9 & 221.9 & 0.6 & $91 \pm 81$ & 126.9 & 221.9 & 0.6 \\
\hline W2 & $362.3 \pm 69$ & 367.9 & 189.3 & 1.9 & $315 \pm 66$ & 355.9 & 132.5 & 2.7 & $377.6 \pm 89$ & 700.4 & 463.9 & 1.5 & $315 \pm 66$ & 355.9 & 132.5 & 2.7 \\
\hline W3 & $657 \pm 148$ & 709.1 & 451.5 & 1.6 & $610.8 \pm 63$ & 661.6 & 257 & 2.6 & $692.9 \pm 136$ & 719 & 352.2 & 2 & $610.8 \pm 70$ & 672.2 & 292.2 & 2.3 \\
\hline W4 & $1227 \pm 171$ & 1020 & 476.7 & 2.1 & $794 \pm 80$ & 676 & 264 & 2.5 & $1145 \pm 169$ & 824 & 414 & 2 & $849.5 \pm 130$ & 735.4 & 393.1 & 1.9 \\
\hline W5 & $1923.8 \pm 229$ & 1269 & 915 & 1.4 & $1294.7 \pm 114$ & 737.6 & 410 & 1.8 & $1739.9 \pm 208$ & 947 & 658.4 & 1.4 & $1379.15 \pm 465$ & 740 & 528 & 1.4 \\
\hline
\end{tabular}

\subsection{Macroscopical findings .}

a. control group and treated after infection showed no internal organs lesions at all intervals

b. OE at $1^{\text {st }}$ slaughtered day ( $3^{\text {rd }}$ dpi) showed slight turbid air sac, slight congested intestinal blood vessels with developing of signs at $6^{\text {th }}$ dpi till $21^{\text {th }}$ dpi pericarditis, air sacculitis, necrotic foci on liver surface, enteritis and straw colored fluid on abdominal cavity while in $\mathbf{S E}$ as $\mathbf{O E}$ group with somewhat high degree especially hepatitis, pericarditis and necrotic foci on liver surface .

c. In POE group showed no pm lesions in contrast of PSE group showed pericarditis, air sacculitis, necrotic foci on liver surface and enteritis started at $9^{\text {th }}$ dpi with absences of signs before that .

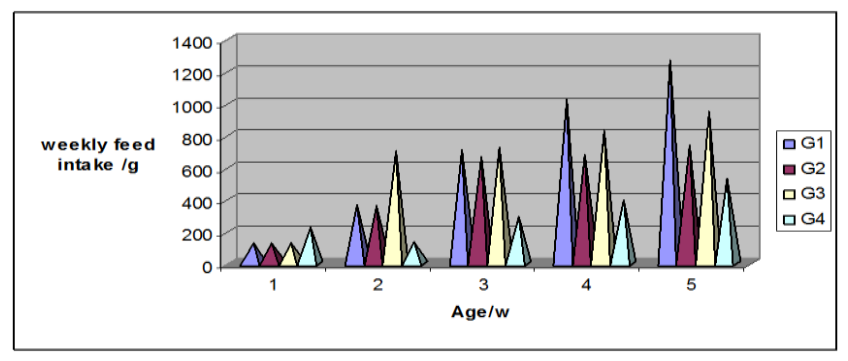

Fig (3): Weekly feed consumption/g in different subcutaneously inoculated groups. G1-negative control group G2-infected untreated group G3-probiotic treated group before infection G4-Antibiotic treated group after infection

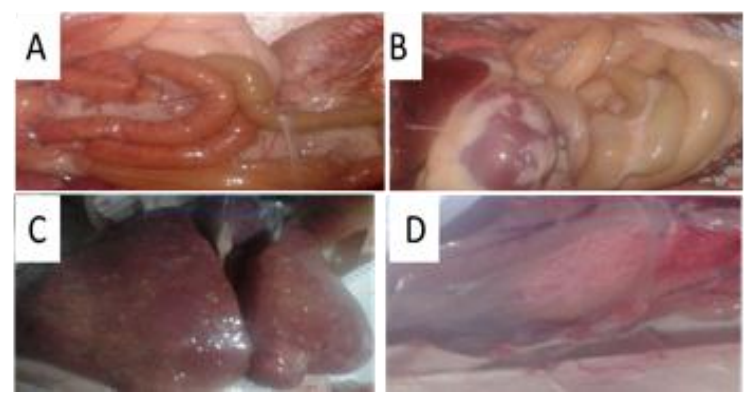

Fig4. Effect of induced $E$. coli infection on chicks

(A) Enteritis of orally infected group, (B) Normal intestine of probiotic treated groups, (C) Necrotic foci on liver surface of subcutaneous infected group, (D) Turbid air sac of $E$. coli infected groups.

\subsection{Microscopical findings}


Photomicrography of both hepatic and intestinal sections in control group showed normal hepatic histology and intestinal mucosa and sub mucosa plates $(1 \mathrm{E} \& 2 \mathrm{E})$ orally un treated group hepatic tissue showed fibrosis on portal area, leucocytic infiltration specially neutrophils and hemolysis

with thrombosis of blood vessels plate (1A) while photomicrography of intestinal sections showed shortening and fusion of villi and infiltration of inflammatory cells , congestion and oedema in serosa plate (2A)

While in subcutaneously inoculated group microphotography of hepatic sections showed showed cirrhosis around portal area, sever congestion and mild leucocytic infiltration plate (1B) while the intestinal villi appeared as club shape with leucocytic infiltration plate (2B).

Orally treated group microphotography of hepatic sections showed very few aggregation of leucocytes in portal area plate (1F) while microphotography of intestinal sections showed normal intestinal villi without signs of inflammation plate $(2 \mathrm{~F})$.

\section{Subcutaneously treated group:}

Microphotography of hepatic sections showed nearly normal portal area without leucocytic infiltration plate (1G) while microphotography of intestinal sections showed normal villi without signs of inflammation plate $(2 \mathrm{G})$.

In probiotic before orally infection microphotography of hepatic sections showed nearly normal portal area without inflammatory cells infiltration plate (1C) while intestinal sections microphotography showed shortening and thickening of intestinal villi due to leucocytic infiltration without signs on inflammation plate $(2 \mathrm{C})$.

But in probiotic before subcutaneously infection: hepatic sections showed mild to moderate leucocytic aggregations on portal area with bile duct hyperplasia plate (1D). The microphotography of intestinal sections showed elongation and thickening of villi due to infiltration with inflammatory cells together with mild inflammation indicated by mild congestion and oedema plate (2D).

\section{Discussion}

E. coli $\mathrm{O}_{125}$ was selected for pathogenicity test due to high virulence on cong red medium and had high frequency in serogrouping in our previous study so we were interested in knowing its pathogenicity in chicks. which un treated groups signs were dullness, depression, dropping of wings, off food, ruffled feather and gradually developed to greenish brown diarrhea, at later stage arthritis, ralls, sneezing and coughing these signs nearly similar to the general signs of E. coli infection recorded by (Awaad, 1972). The performance parameters revealed that was significantly lower in weekly body weight, lower weekly feed consumption weekly body weight gain and bad FCR these results agreed with (Kamel, 2011, Asmaa, 2013).

In the groups which treated with probiotic and groups which treated with antibiotics, The clinical sings and the postmortem lesions were significantly declined and there were significant improvement in the body performance parameters showing high weekly body weight, feed consumption, body weight gain and FCR, these results were similar with (Haghighi et al., 2005, Levkut et al., 2009, Hanaa, 2010, Kamel, 2011, Hussein et al., 2011, Asmaa, 2013), Table 2. Improvement in the histopathological sections of intestine and liver is due to the effect of probiotic and those data is familiar with (Hussein et al., 2011)

In conclusion

The current study demonstrated the high prevalence of E. coli in broiler farms . Experimental infection using selected strain confirmed its ability to induce pericarditis, perihepatitis and airsacculitis . Infection caused low feed consumption, average body weight, weekly body weight gain and FCR. Measures are needed to control $E$. coli infection in poultry farms with using probiotics to avoid and overcome multidrug resistance to reduce economic losses. 

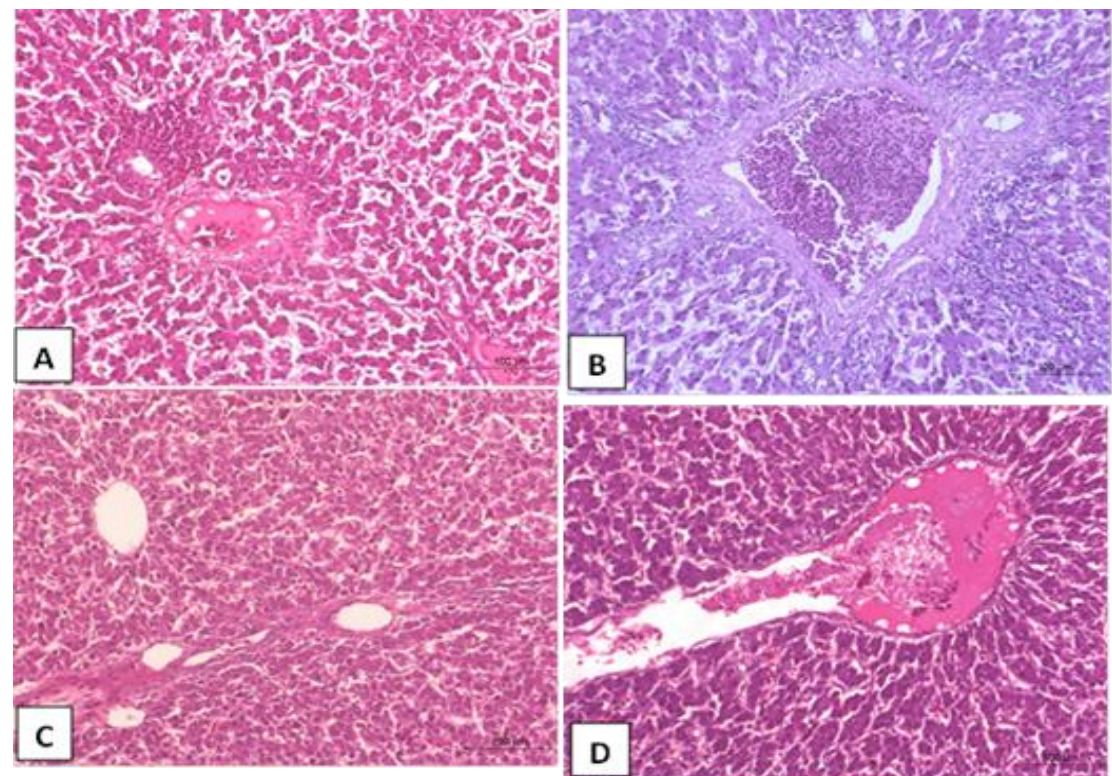

B
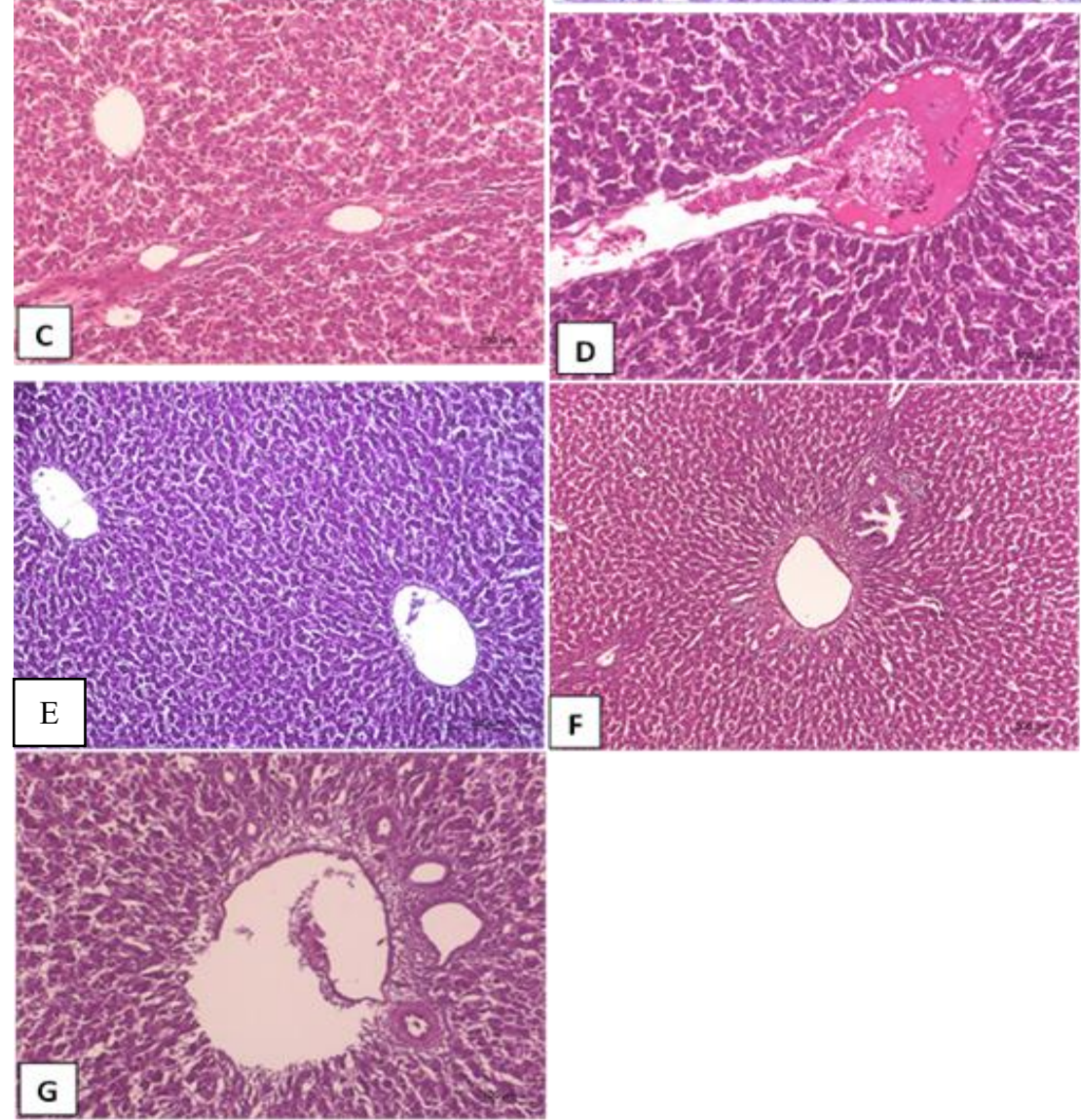

Plate1: Photomicrography of hepatic tissue:

\section{Liver sections of different groups stained with H\&E}

A. orally infected group showing fibrosis on portal area ,leucocytic infiltration specially neutrophiles and hemolysis with thrombosis of blood vessels . B. subcutaneous infected group showed cirrhosis around portal area, sever congestion and mild leucocytic infiltration C. probiotic before orally infection showed nearly normal portal area without inflammatory cells infiltration D. probiotic before subcutaneous infection showed hemolysis and thrombosis of blood vessels from $E$ coli hemolysis $E$. control group showing nearly normal central vein and lymphatic cord F. Orally infected with treatment group showed very few aggregation of leucocytes in portal area without inflammatory signs G. subcutaneous inoculated with treatment group showed nearly normal portal area without leucocytic infiltration. 


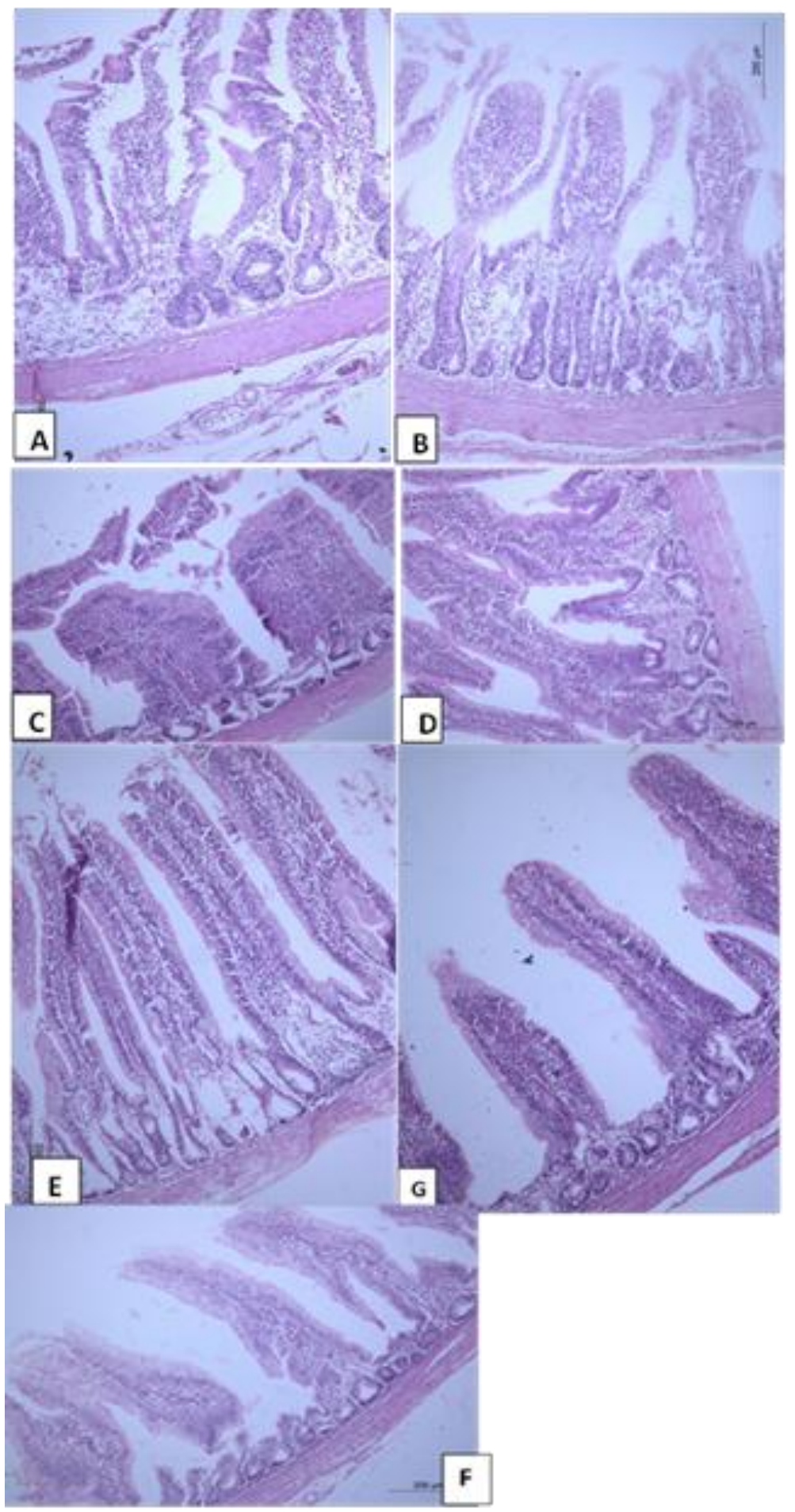

Plate 2 : Photomicrography of intestinal sections:

\section{Intestinal sections of different infected groups}

A. Orally infected group showed sever haemorrhage, congestion and oedema in serosa, shortening and fusion of villi with infiltration of inflammatory cells in submucosa and villarlactel B. Subcutaneous infected group showing club shape with leucocytic infiltration of intestinal villi . C.Probiotic before orally infected group showing Shortening and thickening of intestinal villi due to leucocytic infiltration without signs of inflammation.D. Probiotic with subcutaneous infected group showing mild inflammation indicated by mild congestion and oedema together with elongation and thickening of villi due to infiltration with inflammatory cells. E. control group showing normal elongated villi structure without inflammation F. orally infected group with treatment showing normal villi without signs of inflammation G. subcutaneous infected group with treatment showing nearly normal villi without signs of inflammation. 


\section{References}

Asmaa A.M.M.(2013). Studies on enterobacteriacae in chicken eggs. M.V.Sc. thesis (Poultry diseases dep), Fact. Vet. Med., Beni-Suef Univ. Avian Diseases, 34 (1990), pp. 531-538

Awaad, M. H. (1972) Studies on E. coli infection in chickens M. V. Sc. Thesis, Fac. Vet. Med., Cairo Univ., Egypt.(poultry Sci.

Bancroft J.D., A. StevensTheory and Practice of Histological Technique Churchl Livingstone, Edinburgh (1996), p. 766

Barnes, H.J., Vaillancourt, J.-P. and Gross, W.B. (2003). Colibacillosis. In Y.M. Saif, H.J. Barnes, J.R. Glisson, A.M. Fadley, L.R. McDougald and D.E. Swayne (Eds.), Diseases of Poultry 11 th edition ,Ames Iowa State University Press. 631-652.

Bopp, C. A.; Brenner, F. W.; Wells, J. G. and Strockbine, N. A. (2005). Escherichia, Shigella and Salmonella. In: Manual of Clinical Microbiology.

Dho-Moulin, M. and Fairbrother, J. M. (1999). Avian pathogenic Escherichia coli APEC. Vet. Res. 30 299-316.

Gomis, S. M.; Riddell, C.; Potter, A. A. and Allan, B. J. (2001). Phenotypic and genotypic characterization of virulence factors of Escherichia coli isolated from broiler chickens with simultaneous occurrence of cellulitis and other colibacillosis lesions. Can. J. Vet. Res. 65: 1-6.)

Haghighi H.R., J. Gong, C.L. Gyles, M.A. Hayes, B. Sanei, P. Parvizi, H. Gisavi, J.R. Chambers, S. Sharif Modulation of Antibody-Mediated Immune Response by
Probiotics in Chickens Clin. Diagn. Lab Immunol., 12 (12) (2005), pp. 1387-1392

Hanaa, S. F. (2010). the relationship between caecal coccidiosis and Escherichia coli infection in chicken. M.V.Sc. thesis (poultry), Fact. Vet. Med., Beni Suef Univ.

Hussein,B.M.; El-Hamamy,M.M; Amina, Rania, A. Dessouki (2011). Pathological study on the effect of probiotic in chickens infected with E.Coli.SCVMJ, XVI (2) 2011-177.

Kamel,M.F.(2011). Bacteriological and immunological studies on bacterial causes of enteritis in broilers. M.V.Sc. thesis (Bacteriology, Mycology and Immunology), Fact. Vet. Med., Beni-Suef Univ.

Kim J.H., K.S. Kim Hatchery hygiene evaluation by microbiological examination of hatchery samples ActaVeterinaria Hungarica., 57 (1) (2009), pp. 13-24

LevkutM. , J. Pistl, A. Laukova, V. Revajova, R. Herich, Z. Sevcikova, V. Stromp-ova, R. Szaboova, T. Kokincakova Activity of Enterococcus faecium EF55 against Salmonella Enteritidis in chicks

Nawaz, M. Abbas Irshad, Mubarak Ali* and Ahsan-ul-Haq (2016). Effect of probiotics on growth performance, nutrient digestability and carcass characteristics in broilers The Journal of Animal \& Plant Sciences, 26(3): 2016, Page: 599-604.

Shaw D.P. , D.A. Halvorson Early chick mortality associated with rupture of the yolk sac Avian Diseases, 37 (1993), pp. 720-723

Vidotto M.C., E.E. Muller, J.C. de Freitas, A.A. Alfieri, I.G. Guimaraes, D.S. Santos Virulence factors of avian Escherichia coli 1990. 